\title{
Color Mapping Function
}

National Cancer Institute

\section{Source}

National Cancer Institute. Color Mapping Function. NCI Thesaurus. Code C85844.

A function that transforms the colors of one (source) image to the colors of another (target) image. 